\title{
Effect of V-Ti Addition on Microstructure Evolution and Mechanical Properties of Hot-Rolled Transformation-Induced Plasticity Steel
}

\author{
Hai-Cun $\mathrm{Yu}^{1} \cdot$ Zhao-Zhen Cai $^{1} \cdot$ Gui-Qin Fu' ${ }^{1}$ Miao-Yong Zhu ${ }^{1}$ \\ Received: 11 May 2018/Revised: 2 July 2018/Published online: 16 August 2018 \\ (C) The Chinese Society for Metals and Springer-Verlag GmbH Germany, part of Springer Nature 2018
}

\begin{abstract}
In order to reveal the effect of $\mathrm{V}-\mathrm{Ti}$ addition on the microstructure evolution and the mechanical properties of hot-rolled transformation-induced plasticity (TRIP) steel, two steels with $0.072 \mathrm{~V}-0.051 \mathrm{Ti}$ steel (Bear-V-Ti steel) and $0.001 \mathrm{~V}-$ $0.001 \mathrm{Ti}$ steel (Free-V-Ti steel) were designed, respectively, and the comparison analyses were carried out by performing thermodynamic calculation and an experiment. With the thermodynamic calculation, the critical annealing temperature of a large fraction of retained austenite $(\sim 51 \%)$ obtained via solute enrichment was determined, and an optimized quenching at $650{ }^{\circ} \mathrm{C}$ and tempering at $200{ }^{\circ} \mathrm{C}$ adopted on the as-hot-rolled steel. The results show that the V-Ti TRIP steel displays more optimum mechanical stability during the tensile deformation, since the fraction and the mechanical stability of retained austenite are improved and the microstructure is also ultrarefined by $\mathrm{V}-\mathrm{Ti}$ alloy precipitation. The yield strength of Bear-V-Ti steel increases from 650 to $800 \mathrm{MPa}$, and the ductility reaches 37\%, showing that the comprehensive mechanical properties are greatly improved.
\end{abstract}

Keywords Medium manganese steel $\cdot$ Microstructure evolution $\cdot$ Mechanical properties $\cdot$ V-Ti

\section{Introduction}

The third-generation automotive steel requires excellent mechanical properties, such as high tensile strength and superior plasticity [1]. Since the transformation-induced plasticity (TRIP) steel has the excellent combination of high specific strength and ductility, it has been regarded as a potential candidate for automotive applications [2, 3]. In order to improve the mechanical properties, some previous works investigated the influence of alloying precipitation on microstructures and mechanical properties, especially in Ti/Nb-bearing steels [4-6], Ti/Mo-bearing steel [7] and $\mathrm{V} / \mathrm{Nb}$-bearing steels $[8,9]$. It was proposed that $\mathrm{TiN}$ particles pin at the austenite grain boundary so the grain was refined [10]. The fine grain microstructure can enhance the strength and toughness of steels significantly [11-13].

Available online at http://link.springer.com/journal/40195

Zhao-Zhen Cai

caizz@smm.neu.edu.cn

1 School of Metallurgy, Northeastern University, Shenyang 110819, China
Medium manganese steel is a typical TRIP steel that has received significant attention in recent years. However, most previous works focused on the influences of the manganese and carbon contents on the microstructure evolution and mechanical properties [14-17]. The influence of the alloyed elements, such as $\mathrm{V}$ and $\mathrm{Ti}$, on the properties of medium manganese steel is seldom studied. In the present work, in order to reveal the influence of $\mathrm{Ti}-\mathrm{V}$ alloy elements on the microstructure evolution and improving mechanical properties of medium manganese steel, two steels with $0.072 \mathrm{~V}-0.051 \mathrm{Ti}$ steel (Bear-V-Ti steel) and $0.001 \mathrm{~V}-0.001 \mathrm{Ti}$ steel (Free-V-Ti steel) were designed. Accordingly, the influences of the V-Ti alloy elements on the retained austenite fraction, mechanical stability, grain size evolution, and work-hardening behavior were investigated by performing thermodynamic calculations and an experiment.

\section{Experimental}

The chemical composition of the experimental TRIP steel is presented in Table 1 . A $55 \mathrm{~kg}$ experimental steel cast ingot was manufactured using a vacuum furnace. The ingot 
Table 1 Chemical composition of two experimental steels $(\mathrm{wt} \%)$

\begin{tabular}{lllllllll}
\hline Steel & $\mathrm{C}$ & $\mathrm{Mn}$ & $\mathrm{Al}$ & $\mathrm{V}$ & $\mathrm{Ti}$ & $\mathrm{P}$ & $\mathrm{S}$ & $\mathrm{N}$ \\
\hline Free-V-Ti & 0.13 & 6.22 & 1.38 & - & - & $\leq 0.04$ & $\leq 0.04$ & 0.004 \\
Bear-V-Ti & 0.13 & 6.24 & 1.37 & 0.072 & 0.051 & $\leq 0.04$ & $\leq 0.04$ & 0.004 \\
\hline
\end{tabular}

was heated at $1200{ }^{\circ} \mathrm{C}$ for $2 \mathrm{~h}$, hot forged to rods with a section size of $100 \mathrm{~mm} \times 30 \mathrm{~mm}$, and then air cooled to the room temperature. In order to promote the solution treatment adequately, the rods were soaked at $1200{ }^{\circ} \mathrm{C}$ for $2 \mathrm{~h}$, and then hot-rolled to $3.5 \mathrm{~mm}$ thickness in the temperature range of $1150-940{ }^{\circ} \mathrm{C}$ and finally quenched to the room temperature.

In order to establish the appropriate heat treatment procedures, the critical temperatures of $A_{\mathrm{c} 1}$ (austenite start temperature) and $A_{\mathrm{c} 3}$ (ferrite finish temperature) of the designed steels were obtained by Thermo-Calc software. In order to reveal the scale of austenite and precipitate strength, a two-stage heat treatment process was adopted. Considering the precipitation behavior of $(\mathrm{V}-\mathrm{Ti}) \mathrm{C} / \mathrm{N}$ in austenite and ferrite, first, the as-hot-rolled sheets were soaked at $600{ }^{\circ} \mathrm{C}, 650{ }^{\circ} \mathrm{C}, 700{ }^{\circ} \mathrm{C}$, and $750{ }^{\circ} \mathrm{C}$ for $60 \mathrm{~min}$, and then, immediately quenched in the water. Next, the quenched samples were tempered at $200{ }^{\circ} \mathrm{C}$ for $20 \mathrm{~min}$ and then air cooled to the room temperature; the tempering not only reduces the internal stress but also improves the stability of austenite, leading to improved ductility [18].

Tensile specimens with a width of $1.25 \mathrm{~mm}$ and a gauge length of $35 \mathrm{~mm}$ were machined from the heat-treated sheets with the tensile axis parallel to the prior rolling direction. Tensile tests were carried out at the room temperature using a universal testing machine (SANSCMT5000) at a constant crosshead speed of $3 \mathrm{~mm} /$ min. The samples were etched with a $25 \%$ sodium bisulfite solution. The microstructure was examined using an optical microscope (OM), a scanning electron microscope (SEM), and a transmission electron microscope (TEM) with energy-dispersive spectrometer (EDS). The volume fraction of austenite was determined by X-ray diffraction (XRD) with $\mathrm{Cu} K_{\alpha}$ radiation using a direct comparison method [19], involving the use of integrated intensities of $(200)_{\alpha}$ and $(211)_{\alpha}$ peaks and those of $(200)_{\gamma},(220)_{\gamma}$, and (311) $)_{\gamma}$ peaks. The volume fraction of the austenite $V_{\mathrm{A}}$ was calculated using the following equation [20]:

$V_{\mathrm{A}}=1.4 I_{\gamma} /\left(I_{\alpha}+1.4 I_{\gamma}\right)$,

where $I_{\gamma}$ is the integrated intensity of $\gamma$-phase and $I_{\alpha}$ is the integrated intensity of $\alpha$-phase.

\section{Results}

\subsection{Thermodynamics-Based Predictions of Intercritical Annealing Temperature}

The equilibrium phase fraction as a function of temperature was obtained from the TCFE7 database in Thermo-Calc, as shown in Fig. 1a. In order to obtain various phase fractions between 20 and $70 \%$, four intercritical annealing temperatures ranging from 600 to $750{ }^{\circ} \mathrm{C}$ were selected. At $650{ }^{\circ} \mathrm{C}$, the fraction of austenite was predicted to be about $52 \%$ at the equilibrium state with cementite completely avoided. A model has been proposed by Moor et al. [21] to predict the fraction of stabilized austenite at room temperature through $\mathrm{C}$ partitioning from ferrite into austenite, based on the following Koistinen-Marburger (KM) equation [22]:

$f_{\alpha}=1-\exp \left[-\alpha\left(M_{\mathrm{s}}-T\right)\right]$,

where $f_{\alpha}$ is the fraction of a thermal martensite formed upon cooling, $M_{\mathrm{s}}$ is the martensite start temperature, $T$ is the quenching temperature, and $\alpha$ is a material constant whose value is 0.011 for many steels regardless of the chemical composition.

The thermal stability of austenite, $M_{\mathrm{S}}$ temperature, can be estimated with the formula proposed in Ref. [23-25]:

$M_{\mathrm{S}}=545-423 x_{\mathrm{c}}-30.4 x_{\mathrm{Mn}}-7.5 x_{\mathrm{Si}}+30 x_{\mathrm{Al}}$

$$
-60.5 V_{\gamma}^{-1 / 3}
$$

where $x_{i}(i=\mathrm{C}, \mathrm{Mn}, \mathrm{Si}, \mathrm{Al})$ is the concentration of the alloying element $i$ and $V_{\gamma}$ is the fraction of vanadium in austenite (wt\%), respectively, The calculated $\mathrm{C}$ and $\mathrm{Mn}$ concentrations in austenite as a function of temperature are given in Fig. 2a, b. The dependency of the $\mathrm{C}$ content on the temperature in Fig. 2a is closely associated with the formation of cementite and its critical dissolution temperature. The $\mathrm{Mn}$ and $\mathrm{Al}$ contents are not obviously different between Free-V-Ti steel and Bear-V-Ti steel. It should be noted that the $\mathrm{V}$ and $\mathrm{Ti}$ microalloying elements combine the $\mathrm{C}$ element in austenite and reduce the thermal stability of retained austenite from 600 to $800{ }^{\circ} \mathrm{C}$ as demonstrated by Thermo-Calc results in Fig. 2a.

After substituting the calculated $M_{\mathrm{s}}$ into Eq. (3), the amount of athermal martensite formed upon cooling can be calculated. Thus, the amount of stabilized austenite at room temperature is predicted in Fig. 2d, by means of the equilibrium austenite fractions in Fig. 1a. The optimal critical 

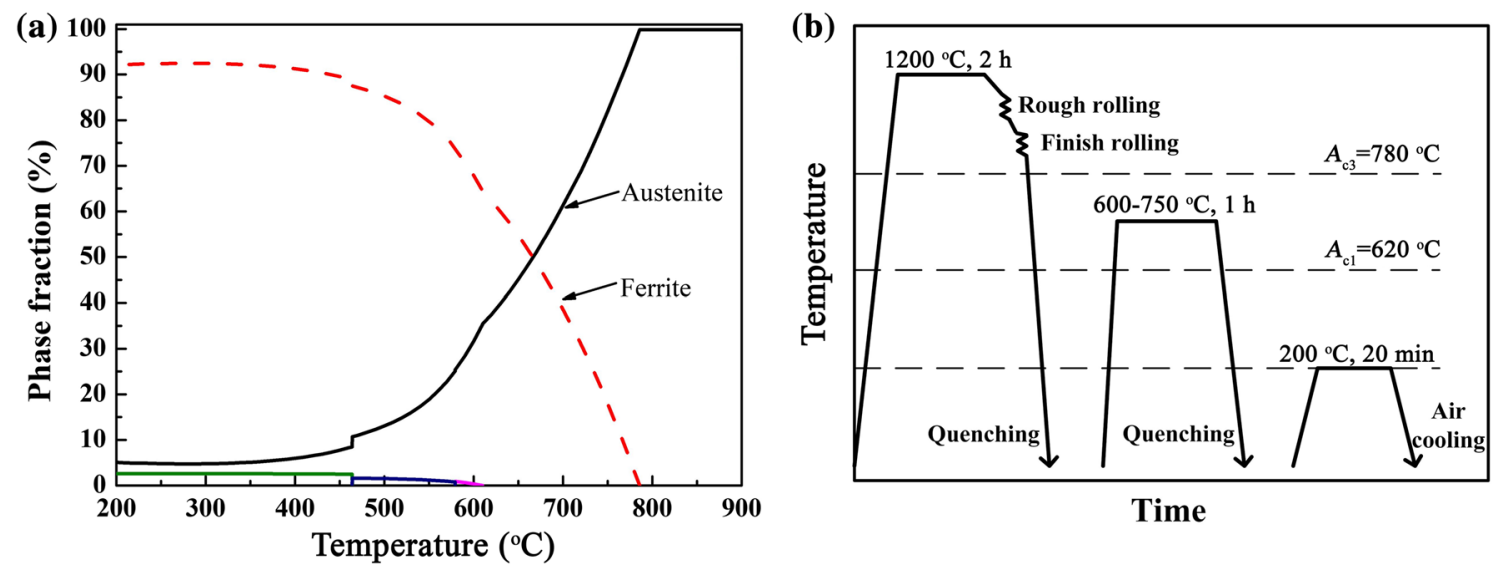

Time

Fig. 1 Phase fraction of experimental steel predicted by Thermo-Calc software a and schematic illustration of heat treatment process $\mathbf{b}$
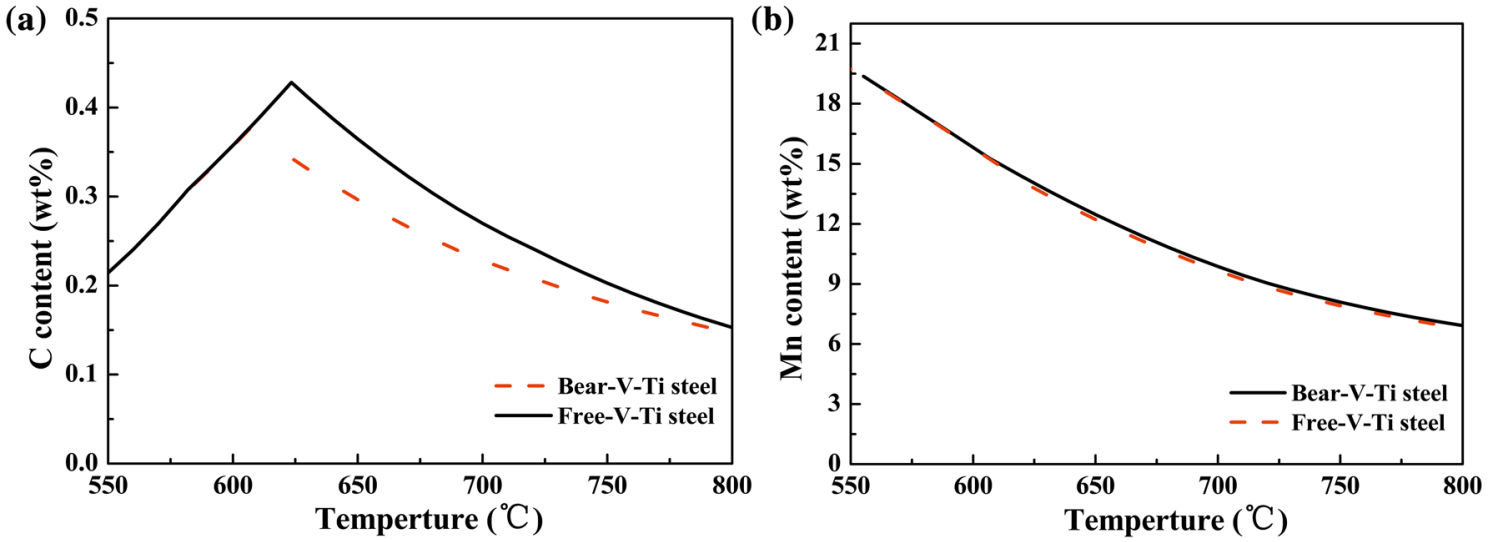

(c)

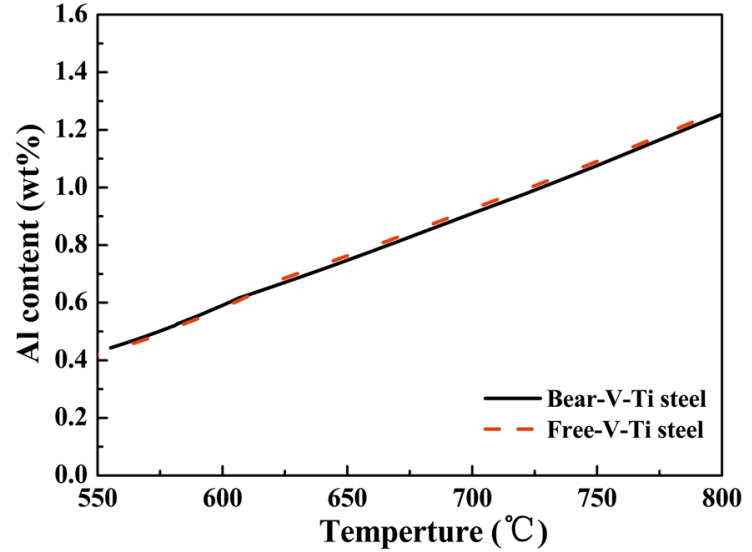

(d)

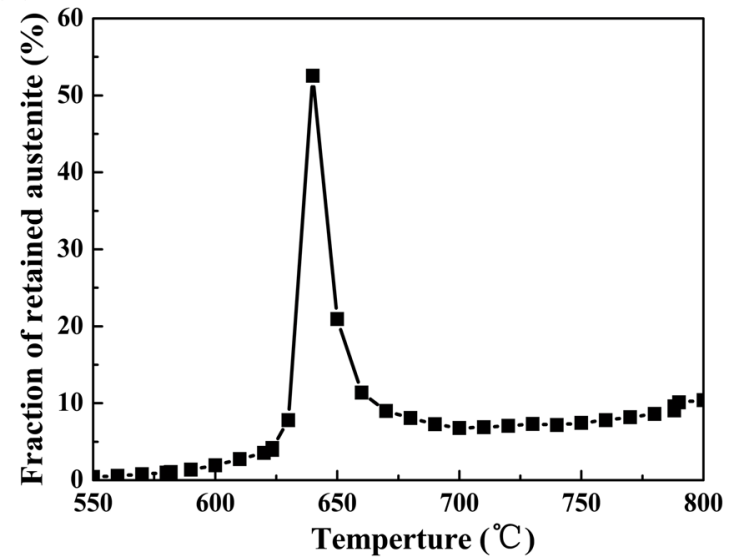

Fig. 2 Thermodynamics calculations for Free-V-Ti steel and Bear-V-Ti steel based on TCFE7 database of Thermo-Calc: a C content in austenite; b Mn content in austenite; c Al content in austenite; d predicted fraction of retained austenite as a function of temperature

annealing temperature was found to be approximately $650{ }^{\circ} \mathrm{C}$, and the predicted maximum austenite fraction for this was about $51 \%$.

\subsection{Microstructure Evolution}

Figure 3a-d shows the SEM micrographs of Free-V-Ti steel quenched at $600{ }^{\circ} \mathrm{C}, 650{ }^{\circ} \mathrm{C}, 700{ }^{\circ} \mathrm{C}$, and $750{ }^{\circ} \mathrm{C}$ and then tempered at $200{ }^{\circ} \mathrm{C}$. The existence of ultra-fine features $(<1 \mu \mathrm{m})$ is evident. The microstructure constituents consisting of ferrite and austenite after quenching at 

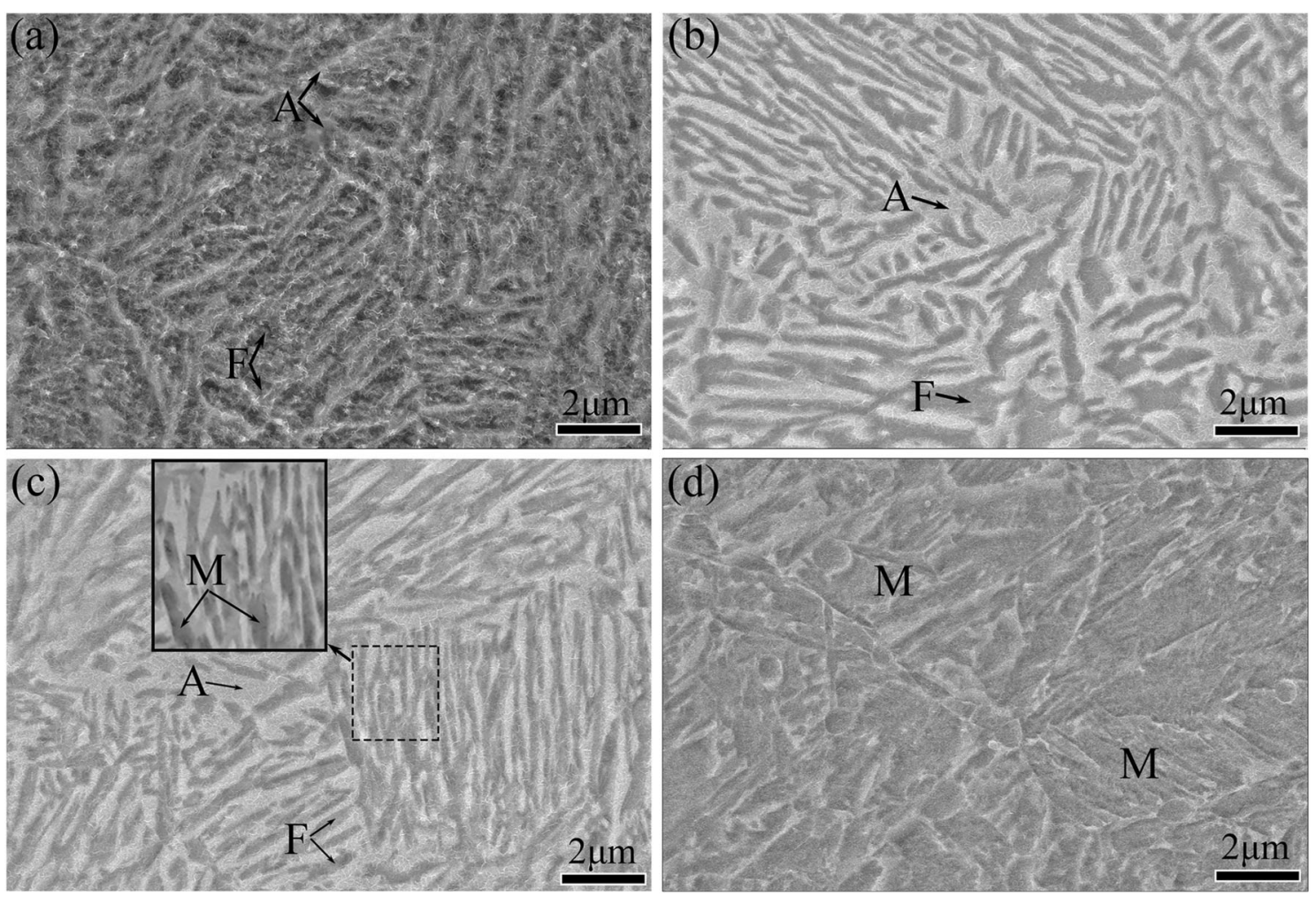

Fig. 3 SEM micrographs of hot-rolled Free-V-Ti steel samples after quenching at different temperatures: a $600{ }^{\circ} \mathrm{C}$; b $650{ }^{\circ} \mathrm{C}$; c $700{ }^{\circ} \mathrm{C}$; d $750{ }^{\circ} \mathrm{C}$
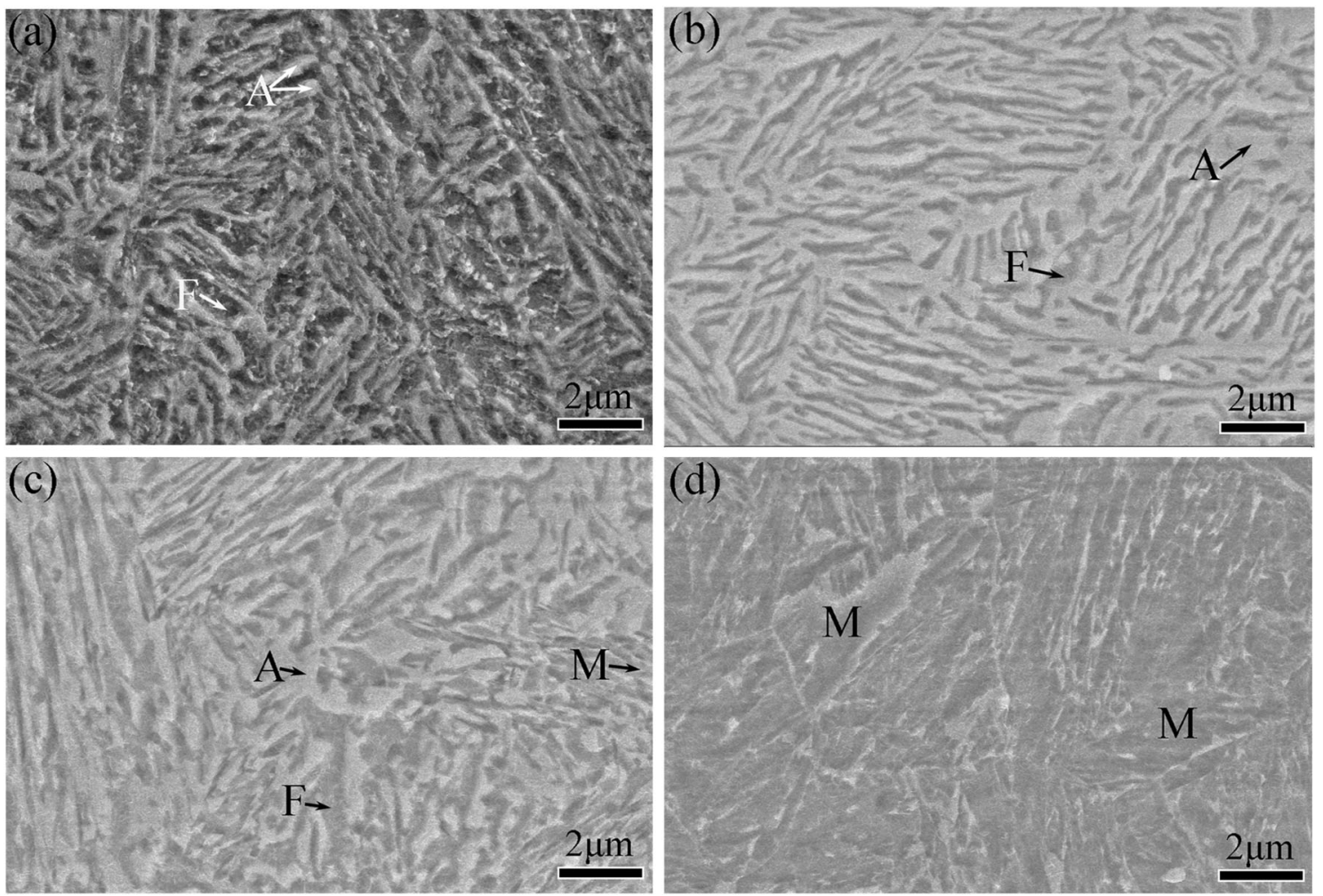

Fig. 4 SEM micrographs of hot-rolled Bear- $\mathrm{V}-\mathrm{Ti}$ steel samples after quenching at different temperatures: a $600{ }^{\circ} \mathrm{C}$; b $650{ }^{\circ} \mathrm{C}$; c $700{ }^{\circ} \mathrm{C}$; d $750{ }^{\circ} \mathrm{C}$ 

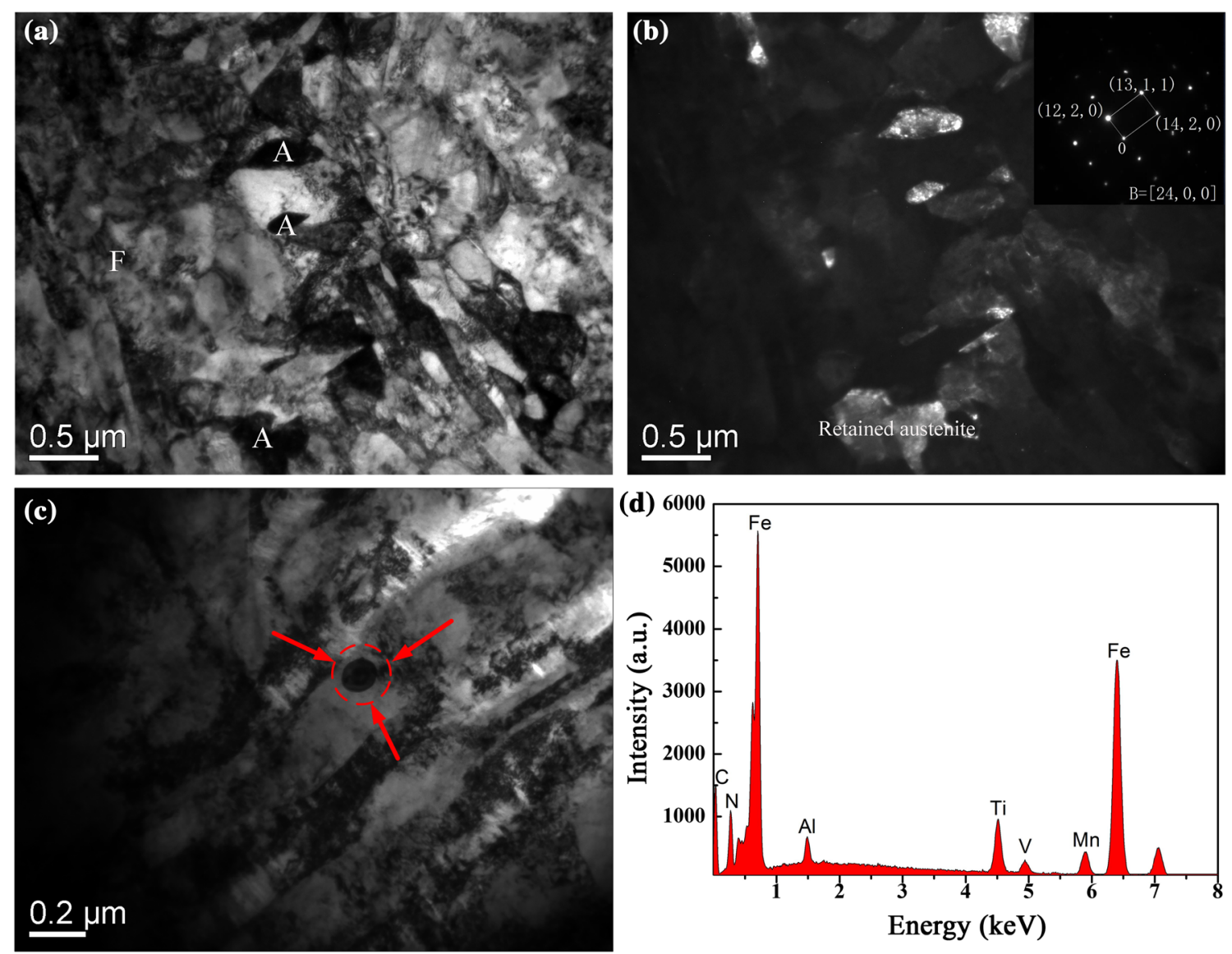

Fig. 5 TEM micrographs of Bear-V-Ti after heat-treated at $650{ }^{\circ} \mathrm{C}$, followed by tempering at $200{ }^{\circ} \mathrm{C}$ : a bright-field image; b dark-field image; c morphologies of precipitates; $\mathbf{d}$ EDS analysis results of small precipitates

$600-650{ }^{\circ} \mathrm{C}$ and tempering at $200{ }^{\circ} \mathrm{C}$ are shown in Fig. 3a, b. When the sample was quenched at $700{ }^{\circ} \mathrm{C}$, martensite (M) appears as indicated in Fig. 3c. As the temperature increases to $750{ }^{\circ} \mathrm{C}$, the austenite was significantly decreased because of extensive martensitic transformation; the examinations suggested that the microstructure consists by three components: ferrite $(\mathrm{F})$, retained austenite $(\mathrm{A})$, and martensite.

Figure 4 shows the SEM microstructures of the hotrolled Bear-V-Ti steel heat-treated at different temperatures. The microstructural constituents are ferrite and retained austenite when the samples were quenched at $600{ }^{\circ} \mathrm{C}$ and $650{ }^{\circ} \mathrm{C}$, respectively. For the samples quenched at $750{ }^{\circ} \mathrm{C}$, the microstructural constituents were ferrite, austenite, and martensite, as shown in Fig. 4d. It can be seen that Bear-V-Ti steel has two types of morphologies: lath and block elements. However, the lath austenite is the main phase. The presence of three constituents in the microstructure and their two types of morphology were also confirmed by TEM observations, as shown in Fig. 5a bright-field image and Fig. 5b dark-field image. The lath austenite is distributed among the ferrite, and the block austenite components are connected to each other. However, the mechanical stability of block austenite is lower than that of lath austenite, as is evident in Fig. 6.

From the observation and analysis of the above micromorphology, it is concluded that Bear- $\mathrm{V}-\mathrm{Ti}$ steel has finer grains and a larger amount of retained austenite than Free$\mathrm{V}-\mathrm{Ti}$ steel. The reason for this is that $\mathrm{V}$ and $\mathrm{Ti}$ induce grain refinement; this improves the thermal stability of the retained austenite and results in lesser amount of austenite transforming into martensite when quenching at the critical temperature.

\subsection{Mechanical Properties}

The mechanical properties of Free-V-Ti steel are illustrated in Fig. 6a, c: The ultimate tensile strength (UTS) increases continuously with increasing temperature up to $750{ }^{\circ} \mathrm{C}$, whereas total elongation (TEL) almost decreases with increasing temperature. For the samples heat-treated at $650{ }^{\circ} \mathrm{C}$, the product of UTS $\times$ TEL reaches the maximum of $33.5 \mathrm{GPa} \%$. A comparison shows that Bear-V-Ti steel (as shown in Fig. 6b, d) has superior mechanical 

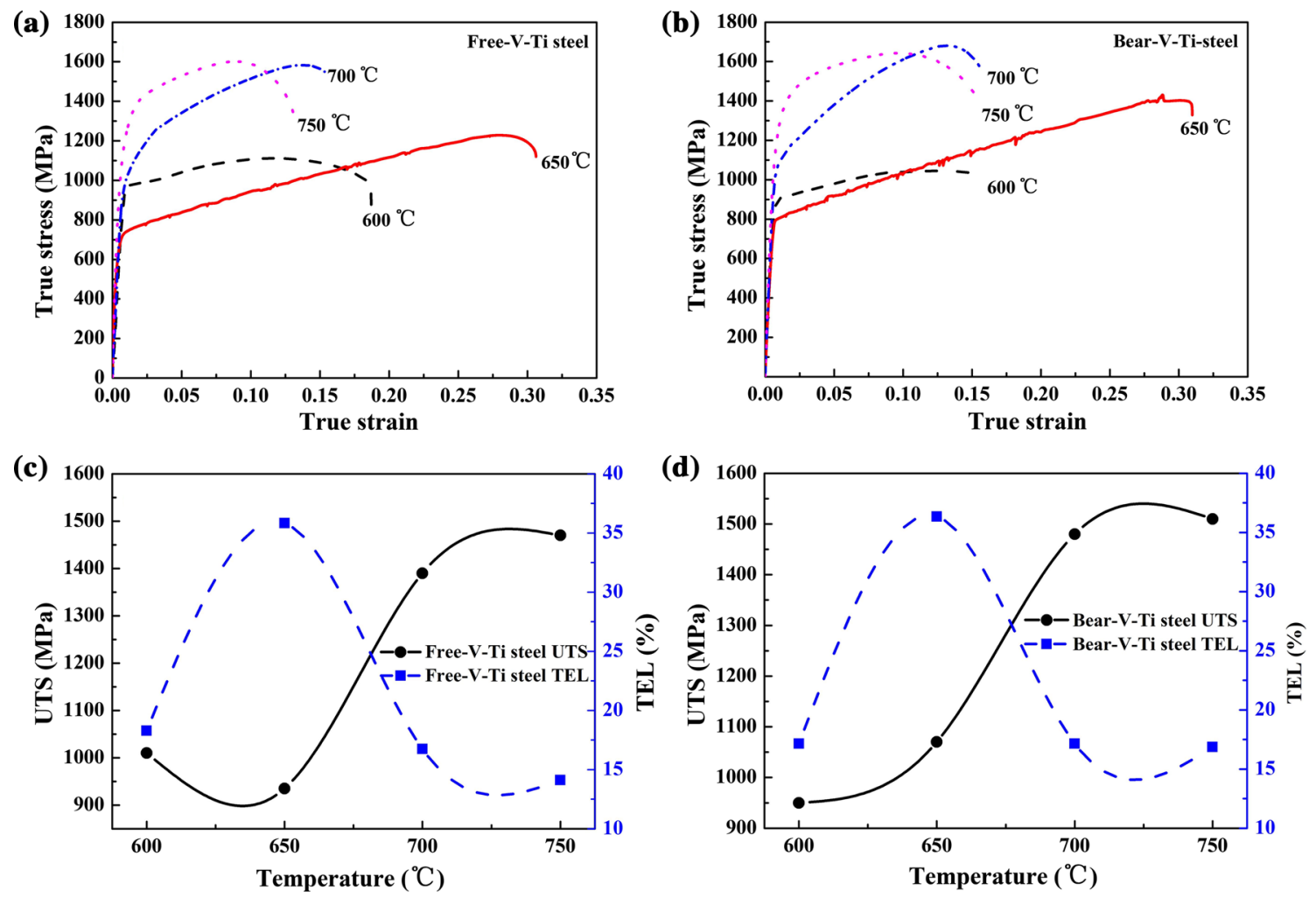

Fig. 6 Mechanical properties of hot-rolled samples: a true strain-stress curves of Free-V-Ti steel; $\mathbf{b}$ true strain-stress curves of Bear-V-Ti steel; c UTS and TEL of Free-V-Ti steel; $\mathbf{d}$ UTS and TEL of Bear-V-Ti steel

properties. From the investigation, it is found that Bear-V$\mathrm{Ti}$ steel has better mechanical properties than Free-V-Ti steel at $650{ }^{\circ} \mathrm{C}$ and that the tensile strength of Bear-V-Ti steel reaches $1070 \mathrm{MPa}$, with a yield strength up to $\sim 800 \mathrm{MPa}$ and elongation similar to that of Free-V-Ti steel.

\subsection{Fraction of Retained Austenite}

Figure 7a, b shows a comparison of XRD patterns of Free$\mathrm{V}-\mathrm{Ti}$ steel and Bear-V-Ti steel undeformed and fracture samples at $650{ }^{\circ} \mathrm{C}$. It is implied that the austenite fraction was reduced considerably after tensile failure. The volume fractions of retained austenite in fractured samples of Free$\mathrm{V}-\mathrm{Ti}$ steel and Bear-V-Ti steel were $18 \%$ and $15 \%$, respectively, and the original amounts of austenite were $53.3 \%$ and $48.5 \%$, respectively (as shown in Fig. 7c). The austenite transformation ratio in Free-V-Ti steel and Bear$\mathrm{V}-\mathrm{Ti}$ steel was $66.03 \%$ and $68.75 \%$, respectively (as shown in Fig. 7d). Thus, this indicates that in Bear-V-Ti steel, significant TRIP effect took place during tensile deformation.

\section{Discussion}

\subsection{Influence of $\mathbf{V}$ and Ti on Flow Stress and Grain Size}

The flow stress of multiphase steel $\left(\sigma_{\mathrm{F}}\right)$ can be estimated using the rule of mixtures proposed in Ref. [26, 27]:

$\sigma_{\mathrm{F}}=f_{\alpha} \sigma_{\alpha}+\left(f_{\gamma}-f_{\mathrm{nm}}\right) \sigma_{\gamma}+\left(f_{\mathrm{m}}+f_{\mathrm{nm}}\right) \sigma_{\mathrm{m}}$

where $\sigma_{\alpha}, \sigma_{\gamma}$, and $\sigma_{\mathrm{m}}$ are the flow stresses of ferrite, austenite, and martensite; $f_{\alpha}, f_{\gamma}, f_{\mathrm{m}}$, and $f_{\mathrm{nm}}$ are the volume fractions of ferrite, austenite, martensite, and newly generated martensite during tensile testing, respectively. In case of Bear-V-Ti steel for example, when the samples are heated in the range of $600-750{ }^{\circ} \mathrm{C}$, the microstructure constituents consist of ferrite and austenite, based on the XRD results (Fig. 7c); further, the austenite fraction and the austenite transformation ratio increase with the temperature, so $\sigma_{\mathrm{F}}$ is dependent on the austenite fraction and TRIP effect. Moreover, due to the TRIP effect, the TEL increases with temperature. For the samples treated in the range of $700-750{ }^{\circ} \mathrm{C}$, the microstructure constituents consist of ferrite, austenite, and martensite as the dominant phases, so $\sigma_{\mathrm{F}}$ depends on the martensite fraction. Because of the increasing martensite fraction, the TEL decreases with temperature. In summary, the UTS increasing with 

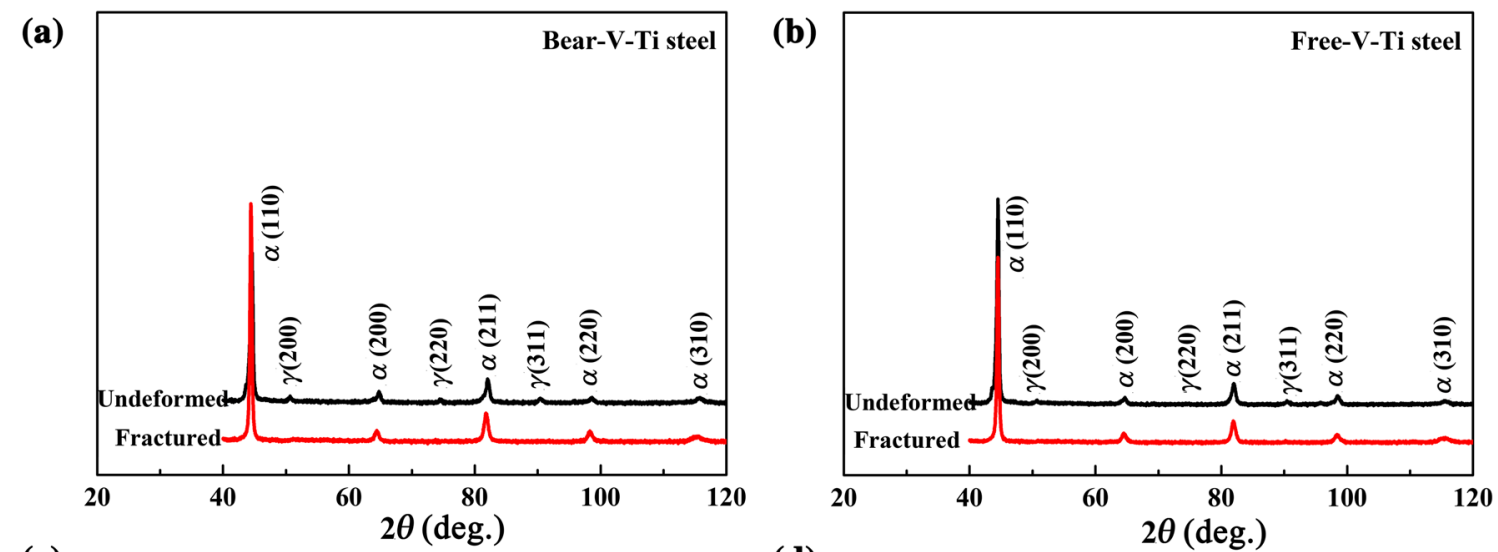

(c)

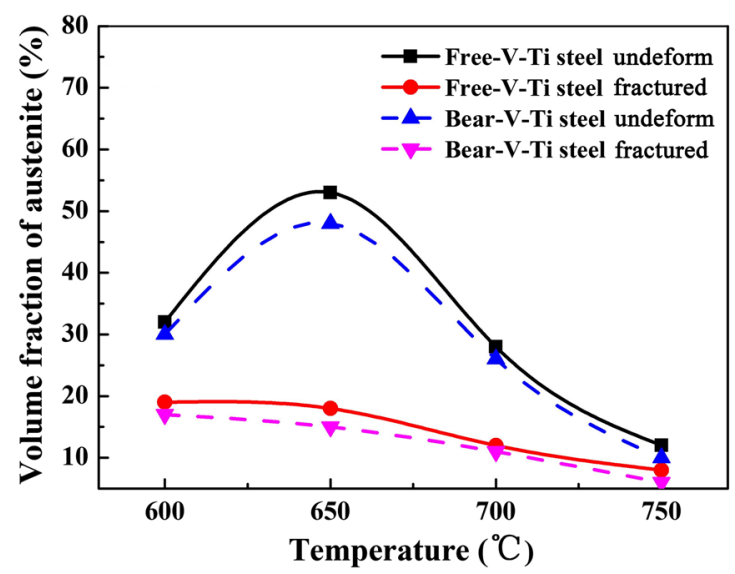

(d)

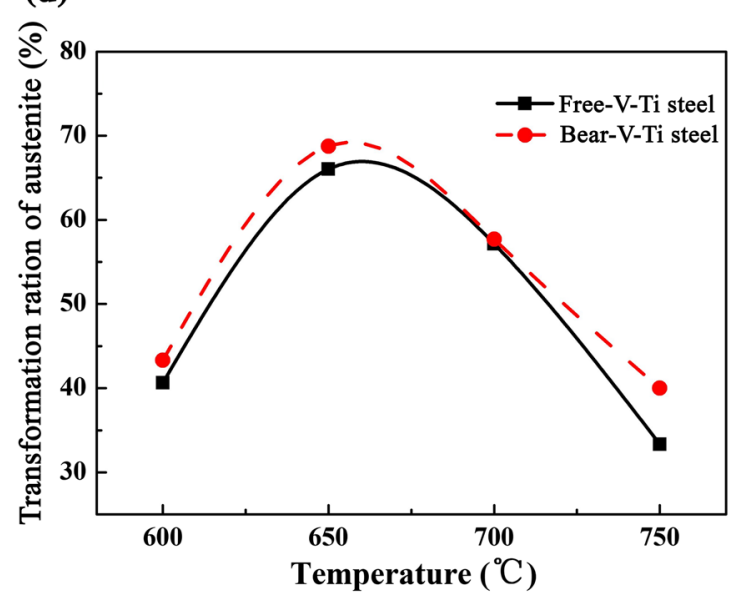

Fig. 7 Measured austenite fractions of undeformed and fractured samples and austenite transformation ratio at $600{ }^{\circ} \mathrm{C}, 650{ }^{\circ} \mathrm{C}, 700{ }^{\circ} \mathrm{C}$, and $750{ }^{\circ} \mathrm{C}$ : a XRD patterns of Bear-V-Ti steel at $650{ }^{\circ} \mathrm{C}$; $\mathbf{b}$ XRD patterns of Free-V-Ti steel at $650{ }^{\circ} \mathrm{C}$; $\mathbf{c}$ measured austenite fractions; $\mathbf{d}$ austenite transformation ratio

temperature is mainly because of the proportion of constituent phases, and the variation of TEL is predicted to be associated with the austenite fraction and TRIP effect, which can be further elucidated by studying the workhardening behavior. In addition, the yield strength increased from 650 to $800 \mathrm{MPa}$ (as shown in Fig. 8a, b), owing to the precipitation of $(\mathrm{V}-\mathrm{Ti}) \mathrm{C} / \mathrm{N}$ pinning the grain boundary, which results in the original austenite grain refinement from 16 to $10 \mu \mathrm{m}$, and the results are summarized in Fig. 8c, d.

\subsection{Precipitation and Work-Hardening Behavior}

True stress-strain plots of Bear-V-Ti steel and Free-V-Ti steel samples are presented in Fig. 9a. It can be seen that the flow curves of the two samples are characterized by serrations, which result in fluctuations in the work-hardening rate. The work-hardening rate results obtained from the tensile tests for Bear-V-Ti steel and Free-V-Ti steel at $650{ }^{\circ} \mathrm{C}$ are presented in Fig. 9b. Both work-hardening curves exhibit two stages: ferrite deformation and increasing work hardening attributed to the TRIP effect. It is obvious that the work-hardening rate of Bear-V-Ti steel is higher owing to the austenite volume fraction and its stability.

Based on the above discussion, we can infer that the different stabilities of austenite in the two samples are clear in Fig. 9b. From the work-hardening curve characteristics, it can be seen that Bear-V-Ti steel has a higher workhardening rate than Free- $\mathrm{V}-\mathrm{Ti}$ steel in the strain range of $0.02-0.06$, and the work-hardening rate tends to decrease beyond 0.06. However, Free-V-Ti steel remains stable from 0.02 to 0.29 . This is due to the following reasons.

Bear-V-Ti steel has a higher amount of retained austenite as shown in Fig. 7c, because the thermal stability of the retained austenite can be improved by refining the grains and making the conversion of retained austenite to martensite difficult in case of quenching at the intercritical temperature. However, it has almost the same ductility as Free-V-Ti steel, because the $\mathrm{V}$ and $\mathrm{Ti}$ can be combined with $\mathrm{C}$ in retained austenite and form (V-Ti)C/N 

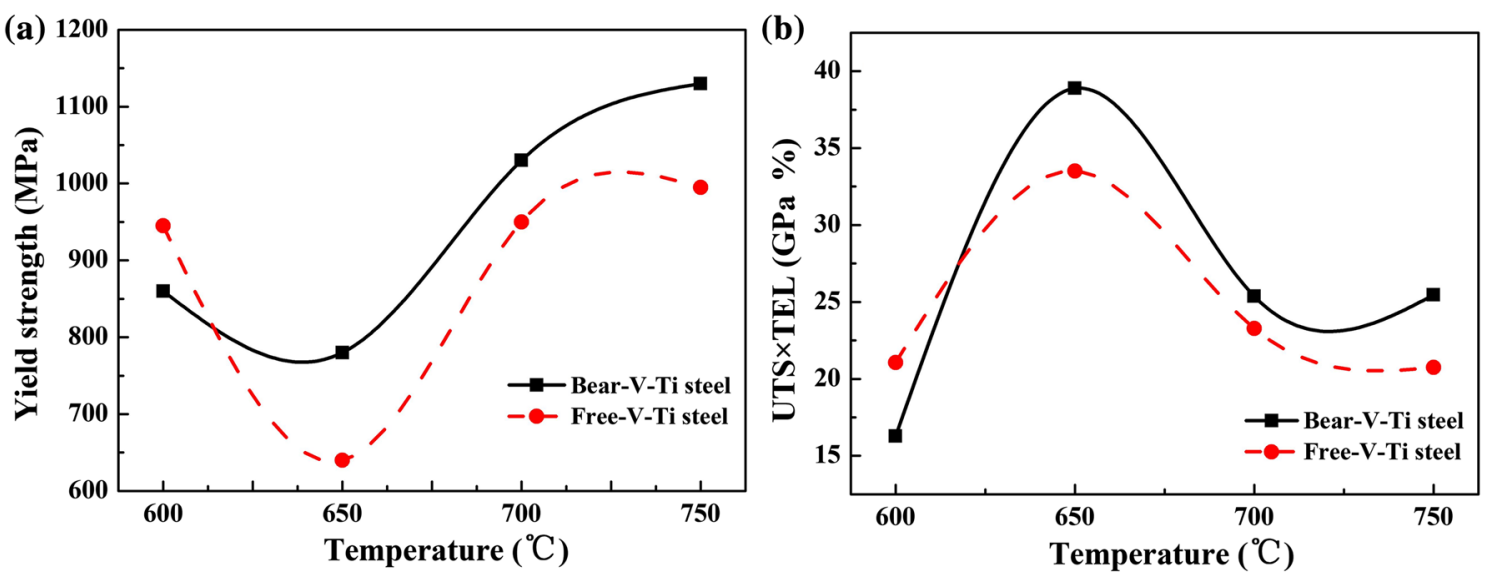

(c)



(d)

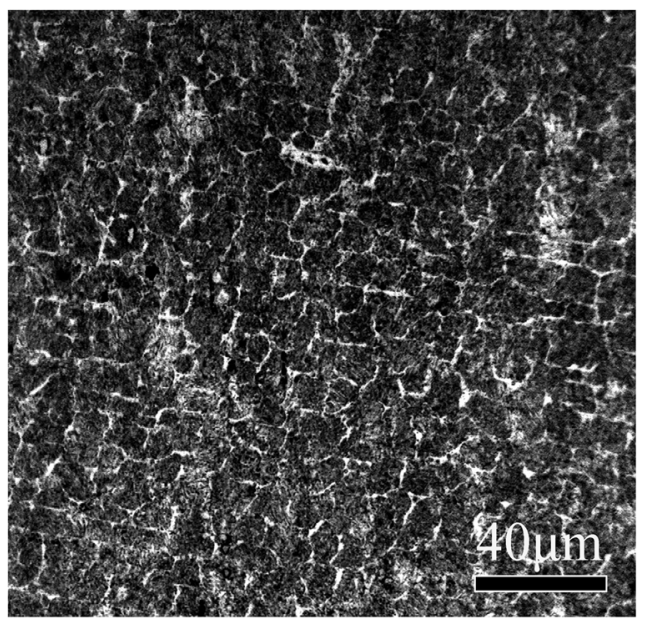

Fig. 8 Tensile properties and micrographs of hot-rolled samples: a yield strength of Free-V-Ti steel and Bear-V-Ti steel; b UTS $\times$ TEL of Free-V-Ti steel and Bear-V-Ti steel; c OM micrograph of Free-V-Ti steel at $650{ }^{\circ} \mathrm{C}$; d OM micrograph of Bear-V-Ti steel at $650{ }^{\circ} \mathrm{C}$
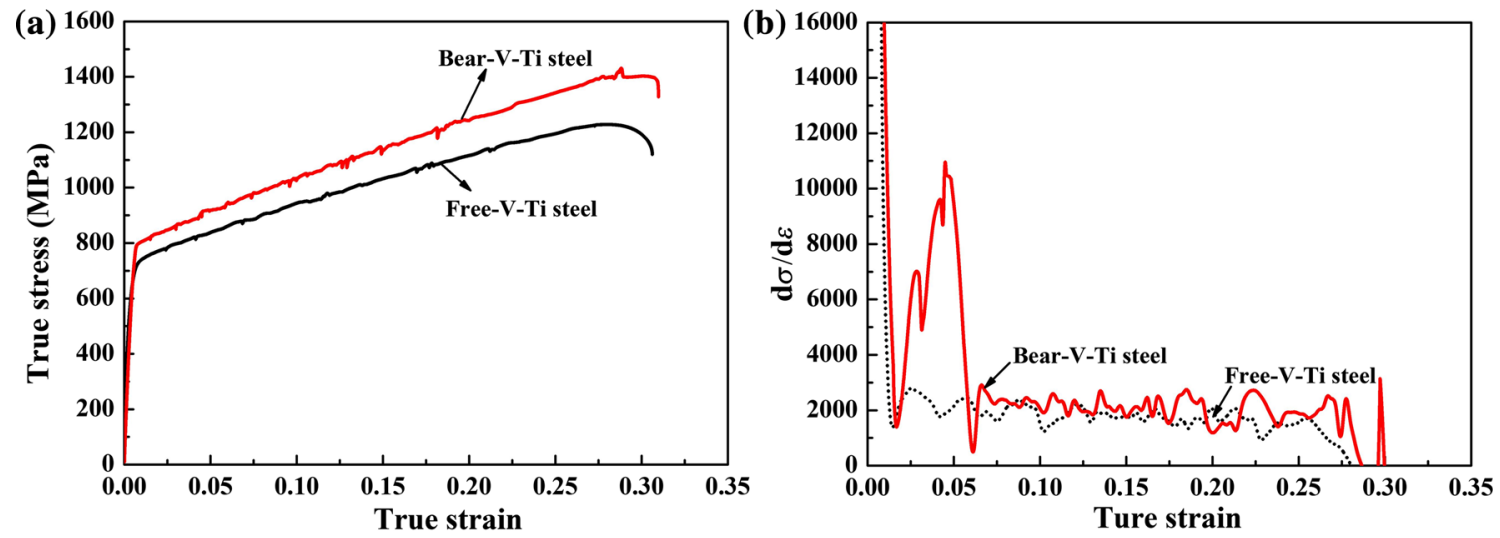

Fig. 9 True strain-stress plot $\mathbf{a}$ and work-hardening rate at $650{ }^{\circ} \mathrm{C} \mathrm{b}$ of Bear-V-Ti and Free-V-Ti steels

precipitate(as shown in Fig. 5c, d) and the loss of $\mathrm{C}$ in retained austenite makes the mechanical stability decline and prompts the TRIP effect to occur at initial strain. Thus, although a large volume fraction of retained austenite is obtained, its lower stability cannot improve the samples' ductility. This conclusion was confirmed by results of a contrast experiment on Bear-V-Ti steel and Free-V-Ti steel in this study. 


\section{Conclusions}

1. The intercritical annealing temperature of $650{ }^{\circ} \mathrm{C}$ was obtained by thermodynamics calculations, and a maximum austenite fraction of about $51 \%$ was achieved. The $\mathrm{C}$ content and thermal stability of austenite in Bear- $\mathrm{V}-\mathrm{Ti}$ steel are higher than those in Free-V-Ti steel.

2. The hot-rolled Bear-V-Ti steel exhibited better mechanical properties than Free-V-Ti steel: The former has TEL of $36.34 \%$, UTS of $1070 \mathrm{MPa}$, and UTS $\times$ TEL of 38.9 GPa \% . Bear-V-Ti steel also shows finer grains and super yield strength due to the precipitation of $(\mathrm{V}-\mathrm{Ti}) \mathrm{C} / \mathrm{N}$ pinning the grain boundary and precipitation strengthening.

3. Bear-V-Ti steel has finer grains compared with Free$\mathrm{V}-\mathrm{Ti}$ steel, but owing to the loss of $\mathrm{C}$ that was combined with $\mathrm{V}$ and $\mathrm{Ti}$ in austenite, the mechanical stability of austenite and the ductility of the steel are lower than those of Free-V-Ti steel.

Acknowledgements This work was supported financially by the National Natural Science Foundation of China (Nos. U1660204 and 51774075) and the Fundamental Research Funds for the Central Universities of China (No. N162504001).

\section{References}

[1] V. Mertinger, E. Nagy, F. Tranta, J. Sólyom, Mater. Sci. Eng. A 481, 718 (2008)

[2] R. Sun, W.H. Xu, C.Y. Wang, J. Shi, H. Dong, W.Q. Cao, Steel Res. Int. 83, 316 (2012)

[3] J. Shi, H.F. Xu, J. Zhao, W.Q. Cao, C. Wang, C.Y. Wang, J. Li, H. Dong, Acta Metall. Sin. (Engl. Lett.) 25, 111 (2012)

[4] G. Xu, X.L. Gan, G.J. Ma, F. Luo, H. Zou, Mater. Des. 31, 2891 (2010)

[5] Z. Jia, R.D.K. Misra, R. O’Malley, S.J. Jansto, Mater. Sci. Eng. A 528, 7077 (2011)
[6] A.I.Z. Farahat, T.A. El-Bitar, Mater. Sci. Eng. A 527, 3662 (2010)

[7] L. Dongyeol, J.K. Kim, S. Lee, K. De Cooman, C. Bruno, Mater. Sci. Eng. A 706, 14 (2017)

[8] S.W. Ooi, G. Fourlaris, Mater. Charact. 56, 214 (2006)

[9] X.L. Li, C.S. Lei, X.T. Deng, Y.M. Li, Y. Tian, Z.D. Wang, G.D. Wang, Acta Metall. Sin. (Engl. Lett.) 30, 1067 (2017)

[10] C.S. Smith, Trans. AIME 175, 15 (1948)

[11] Y. Tomita, K. Okabayashi, Metall. Trans. A 17, 1203 (1986)

[12] R. Lazarova, R.H. Petrov, V. Gaydarova, A. Davidkov, A. Alexeev, M. Manchev, Mater. Des. 32, 2734 (2011)

[13] D. Rasouli, A.S.H. Khameneh, A. Akbarzadeh, G.H. Daneshi, Mater. Des. 30, 2167 (2009)

[14] H.W. Luo, J. Shi, C. Wang, W.Q. Cao, X.J. Sun, H. Dong, Acta Mater. 59, 4002 (2011)

[15] H.J. Jun, O. Yakubovsky, N. Fonstein, Stability of retained austenite in medium Mn TRIP steels. Paper presented at the Proceedings of the 1st International Conference on High Manganese Steels, HMnS2011, Yonsei University, Seoul, Korea, 1-5 January 2011

[16] R.L. Miller, Metall. Trans. A 3, 905 (1972)

[17] M.J. Merwin, Mater. Sci. Forum 4327, 539 (2007)

[18] Z.H. Cai, H. Ding, R.D.K. Misra, H. Kong, H.Y. Wu, Mater. Sci. Eng. A 595, 86 (2014)

[19] K. Sugimoto, B. Yu, Y. Mukai, S. Ikeda, ISIJ Int. 9, 1194 (2005)

[20] N.H. van Dijk, A.M. Butt, L. Zhao, J. Sietsma, S.E. Offerman, J.P. Wright, S. van der Zwaag, Acta Mater. 53, 5439 (2005)

[21] E.D. Moor, D.K. Matlock, J.G. Speer, M.J. Merwin, Scr. Mater. 64, 185 (2011)

[22] D.P. Koistinen, R.E. Marburger, Acta Metall. 7, 59 (1959)

[23] E. Jimenez-Melero, N.H. van Dijk, L. Zhao, J. Sietsma, S.E. Offerman, J.P. Wright, S. van der Zwaag, Scr. Mater. 56, 421 (2007)

[24] E. De, D.K. Moor, J.G. Matlock, M.J.Merwin Speer, Scr. Mater. 64, 185 (2011)

[25] S. Lee, B.C. De Cooman, Metall. Mater. Trans. A 44, 5018 (2013)

[26] J.G. Speer, A.M. Streicher, D.K. Matlock, R. Rizzo, G. Krauss, Quenching and partitioning: a fundamental new process to create high strength TRIP sheet microstructures. Paper presented at the Proceedings of Symposium on Austenite Formation and Decomposition, Chicago, Illinois, 5-22 November 2003

[27] D. Embury, O. Bouaziz, Annu. Rev. Mater. Res. 40, 243 (2010) 